$0092-8240(95) 00018-6$

\title{
CONTRIBUTIONS OF MEMORY B CELLS TO SECONDARY IMMUNE RESPONSE
}

\author{
- SHU-GUANG GUAN \\ Department of Modern Applied Physics, \\ Tsinghua University, \\ Beijing, 100084 \\ P. R. China \\ - AN-SHEN QI \\ Physics Department, \\ Beijing Normal University, \\ Beijing, 100875, \\ P. R. China
}

\begin{abstract}
The secondary immune response is one of the most important features of immune systems. During the secondary immune response, the immune system can eliminate the antigen, which has been encountered by the individual during the primary invasion, more rapidly and efficiently. Both $\mathrm{T}$ and $\mathrm{B}$ memory cells contribute to the secondary response. In this paper, we only concentrate on the functions of memory $B$ cells. We explore a model describing the memory contributed by the specific long-lived clone which is maintained by continued stimulation with a small amount of antigens sequestered on the surfaces of the follicular dendritic cells (FDC). The behavior of the secondary response provided by the model can be compared with experimental observations. The model shows that memory B cells indeed play an important role in the secondary response. It is found that a single memory cell in a long-lived clone may not be longlived. In the present note, the influences of relevant parameters on the secondary response are also explored.
\end{abstract}

1. Introduction. There are three principal characteristics of immune systems: self-nonself recognition, specificity and immune memory. Of these, immune memory is considered to be the key feature and is closely related to the primary and the secondary immune response to the same antigen stimulation. When some kind of antigen insults the immune system for the first time, the immune system would elicit an immune response to eliminate the antigen and this response is referred to as the primary response. If the same antigen insults the immune system again, the system would respond to the antigen with a higher antibody level as well as a faster antigen elimination rate. Such behavior is referred to as the secondary response. In a word, immune systems can remember the antigen having invaded the individual before.

So far, immunologists have got a basic understanding of how the virgin 
B cells can evolve into memory B cells. The microenvironment and signals required for this process have also been investigated (Gray et al., 1990; Klaus, 1978; Liu et al., 1989; Maclennan et al., 1989, 1990; Rajewsky, 1989). So far as we know, three main hypotheses have been proposed to explain the mechanism of immune memory.

The first hypothesis implies that long-lived resting memory B cells account for the memory (Coffman et al., 1977; Hood et al., 1984; Jerne, 1984; Yefenof et al., 1985; Lane et al., 1986; Berek et al., 1987; Levy and Coutinho, 1987; Liu et al., 1988). It assumes that in the primary response, a small number of virgin $B$ cells transform into memory B cells, rather than differentiate into plasma cells. Such memory B cells are long-lived and are at rest with respect to an ordinary time scale. Once the individual encounters the same antigen for the second time, the memory $\mathrm{B}$ cells can be activated and differentiate into plasma cells. Then plasma cells secrete a large amount of antibodies. Thus the secondary response can be elicited. However, this hypothesis is inconsistent with the experimental observations that B-cell memory is short-lived in the absence of antigen (Gray and Skarvall, 1988).

The second hypothesis suggests that immune memory need not be the function of a special type of cell, but rather a property of the system as a whole, "memory can also be carried in the form of interacting networks of cells" (Perelson, 1988, p. 396). Many idiotypic network models have been studied in which immune memory is attributed to the state switches of idiotypic network (Kaufman et al., 1985; De Boer, 1988; Perelson, 1988, 1989; De Boer et al., 1989a,b; Parisi, 1990; Weisbuch, 1990; Sulzer et al., 1993). These models are based on the idiotypic network theory (Jerne, 1974, 1984) which postulates that the immune system can form a complicated interacting network of antibodies and their anti-antibodies. The stimulation of the invading antigen results in the switch of the network from the virgin state into the so-called "immune state". Even after the elimination of the antigen, the immune state can be maintained by the stimulation of the "internal image" of the antigen-antibody whose receptor variable regions mimic epitopes of the antigen (Perelson, 1988; Rajewsky, 1989). Once the same antigen invades the next time, it can be eliminated quickly in the immune state. Thus immune memory can be generated without the continual presence of antigen.

The third hypothesis assumes that the memory resides within a long-lived clone which renews itself by chronic stimulation from the sequestered antigens on FDC (Gray et al., 1986; Gray and Skarvall, 1988; Gray and Leandrerson, 1990; Gray, 1990; Maclennan et al., 1990). Experiments have demonstrated that the follicles, which are found in all second lymphoid tissue, contain a rich network of follicular dendritic cells (FDC). The FDC have been shown to retain protein antigens for a long period after injection (Klaus, 1978; Mandel et al., 1980, 1981; Tew and Mandel, 1978, 1979). After some kind of antigen is 
introduced into the immune system, the specific antibody binds with the antigen and forms an antigen-antibody complex. The majority of these complexes are removed from the immune system by macrophages while a small amount are sequestered on the surfaces of FDC for a long period and act as a continued stimulation source (Klaus, 1978). As a result, immunologists have suggested that "memory does not reside with extremely long-lived resting cells, but rather with extremely long-lived clones that require continual contact with antigen for their survival" (Gray and Leanderson, 1990, p. 14; Gray, 1990). A single $\mathrm{B}$ cell in this clone may not have a long lifespan, but the clone as a whole would be long-lived. The memory generated in this way can be maintained until the sequestered antigen is consumed.

All of the above hypotheses concerning the mechanism of immune memory seem reasonable. However, the third one has acquired more direct experimental evidence. Here we explore a model describing the contributions of momory B cells to the secondary immune response on the basis of the longlived clone hypothesis. Obviously, we do not exclude the other two hypotheses.

2. The Formation of Memory B Cells. Normally, memory B cells are generated during the follicular response which occurs in the primary response to the invading antigen and germinal centers are the birthplace of memory B cells (Klaus, 1978; Coico et al., 1983). B cell follicles are in the resting state except that follicular response occurs. The resting $\mathrm{B}$ follicle comprises a dense FDC network in the central part and also recirculating $B$ cells in the space of the FDC network. After antigen administration, virgin B cells (we do not distinguish virgin B cells from recirculating B cells in this model) are recruited and activated by the antigen. The majority of activated $B$ cells continue to proliferate and differentiate. At last, they become plasma cells which secrete antibodies responsible for the primary response. In the meantime, however, as the "unequal division theory" suggested, a minority of activated B cells give up differentiating into plasma cells and get into the follicles instead (Williamson $e t$ al., 1976). Another "separate lineage theory" suggests that the B cells which have the chance to get into the follicles are from a separate B precursor lineage. This separate $\mathrm{B}$ cell lineage differs from the $\mathrm{B}$ clone which is spccific to the invading antigen and responsible for the production of the primary response (Klinman et al., 1974; see discussion). The reason why a small fraction of activated $B$ cells gets into the follicles rather than differentiates into plasma cells as the majority of the $\mathrm{B}$ cells do is not clear. One possibility suggests that the quantitatively different stimulation accounts for this process while another possibility suggests this process is totally random (Gray and Leanderson 1990; Maclennan et al., 1990). The B cells which have entered the follicles become the B blasts by receiving the stimulating signals (antigen-antibody 
complexes on the surfaces of FDC; C3 and T-helper may also be involved) in the follicular microenvironment (Gray and Leanderson 1990; Klaus, 1978). The appearance of the B blasts marks the beginning of the follicular response. There are three distinct stages in the follicular response. In the first stage, B blasts appear in the FDC network and they proliferate rapidly. Then they become confluent filling the follicle center. The recirculating B cells previously in the FDC network move to the periphery of the follicle forming the follicular mantle. In the second stage, the germinal center forms. This stage of the follicular response is conventionally termed the germinal center reaction. Shortly after B blasts become confluent in the follicle center, centroblasts appear at one pole of the follicle; the area occupied by centroblasts is called the dark zone. Centroblasts proliferate and mutate rapidly. The daughter cells of centroblasts are called centrocytes and they occupy the light zone surrounding the dark zone. Centrocytes also proliferate rapidly. But the majority kill themselves by apotosis if they are not selected by the signals in the follicular microenvironment (Liu, 1989; Maclennan et al., 1990). The centrocytes selected by the signals become memory B cells or plasma cells. These plasma cells differ from normal plasma cells because the former have a relatively long lifespan of about 1 month (Ho et al., 1986; Maclennan et al., 1990), while the latter only have a lifespan of several days. The memory B cells which exit the germinal center face three possibilities: they may enter the recirculating pool, or clonalize new germinal centers, or re-enter the dark zone (Kepler and Perelson, 1993). The germinal center reaction can be maintained until the antigen is eliminated. After the germinal center reaction, the follicular response moves through to the third stage. In this stage, the light and dark zones are no longer apparent; only a central core of B blasts remains.

3. Dynamic Equations. In the model, we assume that a certain antigen is introduced into the immune system twice and it cannot proliferate. A specific antibody type can bind to the introduced antigen and form antigen-antibody complexes. The normal B cells come from bone marrow. When activated by antigen, they proliferate and transform into plasma cells and secrete antibodies. We do not incorporate $T$ cells in the present model. This implies that the $\mathrm{T}$ cell signals, if needed, are always available. Considering how the memory B cells are generated, we mainly accept the "unequal division theory" which assumes the following: during the follicular response, a small part of virgin $\mathbf{B}$ cells get into germinal centers rather than differentiate into plasma cells. In the follicular microenvironment they receive the stimulating signals of the antigen on the surfaces of FDC and undergo an antigen-based selection. In this way, memory $\mathbf{B}$ cells are generated. We further assume that these memory B cells form a long-lived clone (Gray, 1990; Gray and Leanderson, 1990), which the memory $\mathbf{B}$ cells can renew themselves by chronic contact with 
antigens on the surfaces of FDC. The long-lived plasma cells produced in the follicular response are also considered in our model.

According to the consideration stated above, we assume the following.

(1) At the very beginning, i.e. $t=0$, antigen is introduced for the first time to the immune system while at $t=\tau$, the same antigen is reintroduced.

(2) There are $m$ virgin B cells arriving from bone marrow into the recirculating pool every day. Of these, $\alpha \cdot m(0<\alpha<1)$ cells have the chance to get into the FDC network, the other $(1-\alpha) \cdot m$ cells become normal recirculating $\mathrm{B}$ cells. The cells getting into the FDC network still face two evolutionary prospects: $\beta \cdot(\alpha m)(0<\beta<1)$ of them may become $\gamma_{1} \cdot \beta \cdot(\alpha m)$ memory B cells while the rest $(1-\beta) \cdot(\alpha m)$ become $\gamma_{2} \cdot(1-\beta) \cdot(\alpha m)$ long-lived plasma cells after 1 day's proliferation in the germinal center reaction. Here $\gamma_{1}$ indicates that every $\mathrm{B}$ cell in the germinal center during the follicular response gives birth to $\gamma_{1}$ memory $\mathbf{B}$ cclls a day. The meaning of $\gamma_{2}$ is similar. Once the memory $\mathbf{B}$ cells and plasma cells are generated, they enter the recirculating pool.

(3) Because only minute long-lived antigen-antibody complexes persist on FDC (Maclennan et al., 1990), such a small part of complexes are not considered for the antigen elimination in the following model (see equation (4)). Strictly speaking, the generation of memory B cells and long-lived plasma cells in the germinal center reaction is dependent on the selective signals from the sequestered antigen-antibody complexes (also C3 and T-helper) in the follicular microenvironment and is not directly dependent on the invaded antigen concentration. Considering that this selection process is very complicated and the details remain unclear, we do not try to model it explicitly in the present model. We simply consider the germinal center as a "grey box". Though we do not know the precise details, we do know that the virgin B cells which have chance to get into it become memory B cells and long-lived plasma cells when they come out of it. In this model, we assume the quantity of the antigen-antibody complex is always sufficient to generate memory $B$ cells and long-lived plasma cells. Thus given the values of parameter $m, \alpha, \beta, \gamma_{1}$ and $\gamma_{2}$, the generation rates of memory $B$ cells and long-lived plasma cells are two small constants, which are not directly dependent on the antigen concentration (see the first terms on the right of equations (6) and (7)).

(4) We assume that the time needed to develop the memory cells is longer than the period the primary response can maintain. This means that there is no memory $\mathbf{B}$ cell responsible for the primary response to the antigen. This assumption is consistent with experimental observations (Maclennan et al., 1990). In our model, memory B cells proliferate and secrete antibodies immediately after the antigen is introduced the next time at $t=\tau$; before $t=\tau$, the memory $\mathbf{B}$ cells are assumed to be at their developing stage. 
Considering all the definitions and assumptions listed above, we set up the following differential equations:

$$
\begin{gathered}
F(H)=\frac{H}{\theta_{1}+H} \frac{\theta_{2}}{\theta_{2}+H} \\
H=\mathbf{J} \cdot A g \\
\frac{\mathrm{d} A}{\mathrm{~d} t}=S_{\mathrm{N}} F(H) B_{\mathrm{N}}+S_{\mathrm{N}} B_{\mathrm{P}} \mid S_{\mathrm{M}} F(H) B_{\mathrm{M}} \theta(t-\tau)-d_{\mathrm{C}} A H-d_{\mathrm{A}} A \\
\frac{\mathrm{d} A g}{\mathrm{~d} t}=-K \frac{A H}{K_{1}+A} \\
\frac{\mathrm{d} B_{\mathrm{N}}}{\mathrm{d} t}=(1-\alpha) m+P_{\mathrm{N}} F(H) B_{\mathrm{N}}-d_{\mathrm{N}} B_{\mathrm{N}} \\
\frac{\mathrm{d} B_{\mathrm{M}}}{\mathrm{d} t}=\gamma_{1}(\beta \alpha m)+P_{\mathrm{M}} F(H) B_{\mathrm{M}} \theta(t-\tau)-d_{\mathrm{M}} B_{\mathrm{M}} \\
\frac{\mathrm{d} B_{\mathrm{p}}}{\mathrm{d} t}=\gamma_{2}[(1-\beta) \alpha m]-d_{\mathrm{P}} B_{\mathrm{P}}
\end{gathered}
$$

where $A$ and $A g$ are the antibody concentration and the antigen concentration, respectively; $B_{\mathrm{N}}, B_{\mathrm{M}}$ and $B_{\mathrm{P}}$ are the population size of normal $\mathrm{B}$ cells, memory cells and plasma cells, respectively; $m$ is the source rate of virgin B cells; $P_{\mathrm{N}}$ and $P_{\mathrm{M}}$ are the proliferation rates of $B_{\mathrm{N}}$ and $B_{\mathrm{M}}$, respectively; $S_{\mathrm{N}}$ is the antibody secretion rate of B plasma cells evolved from normal B cells while $S_{\mathrm{M}}$ is the antibody secretion rate of B plasma cells evolved from memory B cells; $d_{\mathrm{N}}, d_{\mathrm{M}}$, $d_{\mathrm{P}}$ and $d_{\mathrm{A}}$ are the decay rates of $B_{\mathrm{N}}, B_{\mathrm{M}}, B_{\mathrm{P}}$ and $A$, respectively; $d_{\mathrm{c}}$ is the complex formation rate; $\tau$ indicates the moment at which antigens are introduced to the immune system for the second time; $K$ and $K_{1}$ are constants; $\alpha$ and $\beta$ are the proportion parameters; $\gamma_{1}$ and $\gamma_{2}$ are the increasing constants of $B_{\mathrm{M}}$ and $B_{\mathrm{P}}$ in the germinal center reaction; $F(H)$ is the log-bell shaped activation function (proposed in Perelson and Delisi, 1980; used in De Boer, 1988; De Boer et al., 1989a,b, 1991; Weisbuch et al., 1990; Perelson and Weisbuch, 1992) and its value gives the proportion of normal $\mathbf{B}$ cells or memory $\mathbf{B}$ cells which can be activated to differentiate into plasma cells depending on the field $H . \theta_{1}$ and $\theta_{2}$ are the threshold parameters at which the function approximately reaches its half-maximum, i.e. 0.5 (see Fig. 1). Here we think that the antigen concentra- 


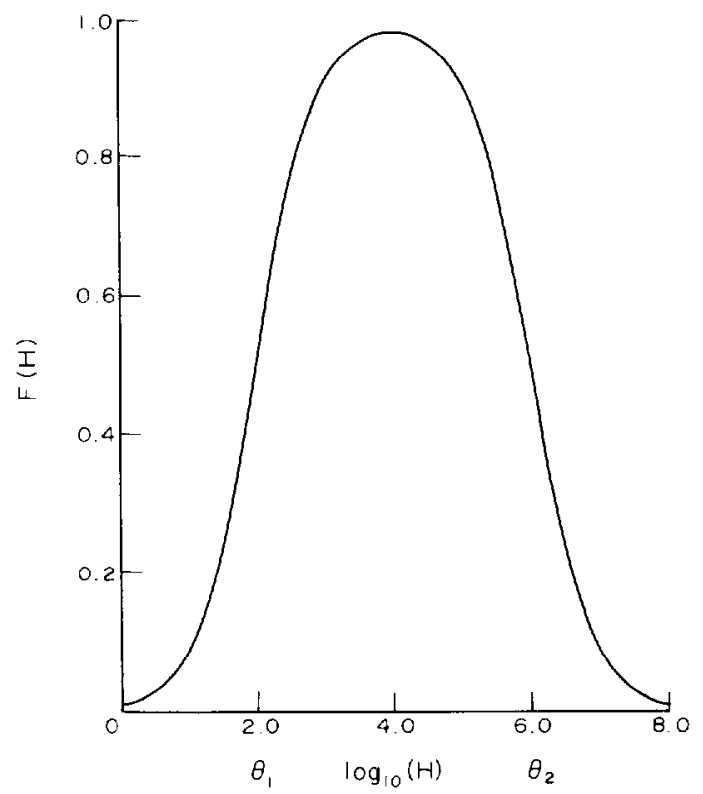

Figure 1. The graph of the log-bell shaped activation function $F(H) . \theta_{1}=10^{2}$, $\theta_{2}=10^{6}$. The maximum of the function is approximately one, and the values of the function at $\theta_{1}$ and $\theta_{2}$ are approximately equal to 0.5 .

tion which is too large suppresses the $B_{\mathrm{N}}$ proliferation rate, i.e. the factor $\theta_{2} /\left(\theta_{2}+H\right)$ in $F(H)$ is suppressive (De Boer et al., 1989b). $\theta(t-\tau)$ is the Heaviside step function: if $t<\tau, \theta(t-\tau)=0$, if $t>\tau, \theta(t-\tau)=1$. As for equation (4), the reader is referred to De Boer et al. (1989b).

4. Parameter Values and Scalings. We define the entire volume of the immune system, for example, the total blood volume of a rat; as the unit volume. The introduced antigen is assumed to be molecular. We use "unit" as the unit of the concentrations of antigen and antibody. One "unit" is equal to the quantity of immunoglobulins which a plasma cell can secrete per day. If a plasma cell secretes $10^{4}$ antibodies $\mathrm{s}^{-1}$ (normally $10^{3}-10^{4}$ ), then one unit is equal to $1.4 \times 10^{-15} \mathrm{~mol}$ (Pcrelson and Weisbuch, 1992); Morcover, the unit of the $B_{\mathrm{N}}$, $B_{\mathrm{M}}$ and $B_{\mathrm{P}}$ population is defined as cell. Considering the definition above, the values of the parameters $\theta_{1}, \theta_{2}, P_{\mathrm{N}}, S_{\mathrm{N}}, d_{\mathrm{A}}, d_{\mathrm{N}}, d_{\mathrm{C}}$ and $J$ are set below (De Boer et al., 1990, 1991; Antia et al., 1991): $\theta_{1}=10^{2}$ unit, $\theta_{2}=10^{6}$ unit, i.e. the cell population expands about $10^{4}$-fold, $m=1.0$ cell $\cdot$ day $^{-1}, P_{\mathrm{N}}=1.0 \mathrm{day}^{-1}$, $S_{\mathrm{N}}=1.0$ unit $\cdot$ cell $^{-1} \cdot \mathrm{day}^{-1}, d_{\mathrm{A}}=0.1 \mathrm{day}^{-1}$ or $0.05 \mathrm{day}^{-1}, d_{\mathrm{N}}=0.5 \mathrm{day}^{-1}$, $d_{\mathrm{C}}=10^{-3}$ unit $^{-1} \cdot \mathrm{day}^{-1}$ and $J=1.0$. Values of $K$ and $K_{1}$ are taken from De Boer et al. (1989b) i.e. $K=1.0$ day $^{-1}, K_{1}=10^{5}$ unit. With regard to the other parameters, $P_{\mathrm{M}}, S_{\mathrm{M}}, d_{\mathrm{M}}, d_{\mathrm{P}}, \alpha, \beta, \gamma_{1}$ and $\gamma_{2}$, we estimate their biologically 
reasonable evaluations as closely as we can. We assume that the memory $B$ cells in the long-lived clone have the same lifespan as those of the normal B cells, i.e. $d_{\mathrm{M}}=d_{\mathrm{N}}$. As to $d_{\mathrm{P}}$, since the long-lived plasma cells generated in germinal center reaction have a lifespan about 1 month (Maclennan et al., 1990), we set $d_{\mathrm{P}}=0.05 \mathrm{day}^{-1} . P_{\mathrm{M}}$ and $S_{\mathrm{M}}$ are the proliferation rate and antibody secretion rate of $B_{\mathrm{M}}$, respectively. They should be larger than that of $B_{\mathrm{N}}$ cells. Then in most cases, we set $P_{\mathrm{M}}=1.5 \mathrm{day}^{-1}, S_{\mathrm{M}}=2.0$ unit $\cdot \operatorname{cell}^{-1} \cdot \mathrm{day}^{-1} . \alpha$ and $\beta$ are the proportion parameters and they are located between 0 and 1 . We set $\alpha=0.2$ and $\beta=0.5 ; \gamma_{1}$ and $\gamma_{2}$ are unknown parameters. Considering that centroblasts and centrocytes proliferated very fast in the germinal center reaction (Maclennan et al., 1990), we set $\gamma_{1}=\gamma_{2}=5.0$.

We now non-dimensionalize equations (1)-(7). Taking the following scalings (De Boer et al., 1990; Perelson and Weisbuch, 1992):

$$
\begin{gathered}
t^{\prime}=t d_{\mathrm{N}}, \quad B_{\mathrm{N}}^{\prime}=B_{\mathrm{N}} \frac{d_{\mathrm{N}}}{m}, \quad B_{\mathrm{M}}^{\prime}=B_{\mathrm{M}} \frac{d_{\mathrm{N}}}{m}, \\
B_{\mathrm{P}}^{\prime}=B_{\mathrm{P}} \frac{d_{\mathrm{N}}}{m}, \quad A^{\prime}=A \frac{d_{\mathrm{N}}^{2}}{m S_{\mathrm{N}}}, \quad A g^{\prime}=A g \frac{d_{\mathrm{N}}^{2}}{m S_{\mathrm{N}}}, \quad H^{\prime}=J A g^{\prime} .
\end{gathered}
$$

Then defining:

$$
\begin{aligned}
\theta_{1}^{\prime}=\theta_{1} \frac{d_{\mathrm{N}}^{2}}{m S_{\mathrm{N}}}, & \theta_{2}^{\prime}=\theta_{2} \frac{d_{\mathrm{N}}^{2}}{m S_{\mathrm{N}}}, & r_{\mathrm{N}}=\frac{p_{\mathrm{N}}}{d_{\mathrm{N}}}, \\
r_{\mathrm{M}}=\frac{p_{\mathrm{M}}}{d_{\mathrm{N}}}, & \delta_{\mathrm{M}}=\frac{d_{\mathrm{M}}}{d_{\mathrm{N}}}, & \delta_{\mathrm{P}}=\frac{d_{\mathrm{P}}}{d_{\mathrm{N}}} \\
\tau^{\prime}=\tau d_{\mathrm{N}}, & \delta_{\mathrm{A}}=\frac{d_{\mathrm{A}}}{d_{\mathrm{N}}}, & S=\frac{S_{\mathrm{M}}}{S_{\mathrm{N}}} \\
\mu=\frac{d_{\mathrm{C}} m S_{\mathrm{N}}}{d_{\mathrm{N}}^{3}}, & K^{\prime}=\frac{K}{d_{\mathrm{N}}}, & K_{1}^{\prime}=\frac{K_{1} d_{\mathrm{N}}^{2}}{m S_{\mathrm{N}}},
\end{aligned}
$$

we obtain:

$$
\begin{gathered}
F(H)=\frac{H}{\theta_{1}+I I} \cdot \frac{\theta_{2}}{\theta_{2}+I I} \\
H=J \cdot A g \\
\frac{\mathrm{d} A}{\mathrm{~d} t}=F(H) B_{\mathrm{N}}+B_{\mathrm{P}}+S F(H) B_{\mathrm{M}} \theta(t-\tau)-\mu A H-\delta_{\mathrm{A}} A
\end{gathered}
$$




$$
\begin{gathered}
\frac{\mathrm{d} A g}{\mathrm{~d} t}=-K \frac{A H}{K_{1}+A} \\
\frac{\mathrm{d} B_{\mathrm{N}}}{\mathrm{d} t}=(1-\alpha)+B_{\mathrm{N}}\left[r_{\mathrm{N}} F(H)-1\right] \\
\frac{\mathrm{d} B_{\mathrm{M}}}{\mathrm{d} t}=\gamma_{1} \beta \alpha+B_{\mathrm{M}}\left[r_{\mathrm{M}} F(H) \theta(t-\tau)-\delta_{\mathrm{M}}\right] \\
\frac{\mathrm{d} B_{\mathrm{P}}}{\mathrm{d} t}=\gamma_{2}(1-\beta) \alpha-\delta_{\mathrm{P}} B_{\mathrm{P}} .
\end{gathered}
$$

In the above equations, for simplicity, we have omitted the superscripts "'”." The steady state of the system reads:

$$
\begin{array}{cc}
\bar{A}=\frac{\gamma_{2}(1-\beta) \alpha}{\delta_{\mathrm{A}} \delta_{\mathrm{P}}}, & A \bar{g}=0, \quad \bar{B}_{\mathrm{N}}=(1-\alpha), \\
\bar{B}_{\mathrm{M}}=\frac{\gamma_{1} \beta \alpha}{\delta_{\mathrm{M}}}, & \bar{B}_{\mathrm{P}}=\frac{\gamma_{2}(1-\beta) \alpha}{\delta_{\mathrm{P}}} .
\end{array}
$$

By stability analysis, it can be seen that the steady state is stable. Using the parameter values given above, the values of the steady state are relatively small (about 10 or so). This indicates that the immune system can return back to the resting state after removal of the antigen. We study the dynamical behaviors of the model by computer simulations. The simulations are processed employing a variable step length Merson routine.

The initial concentration of $B_{\mathrm{N}}$ is $B_{\mathrm{N}}=1.0$; The antibody is set to zero at the beginning; $B_{\mathrm{M}}$ and $B_{\mathrm{P}}$ are produced in immune response since their initial concentrations are zero. The concentration of antigen introduced is located between $\theta_{1}$ and $\theta_{2}$, i.e. between $10^{2}$ and $10^{6}$, which are the suitable antigenic stimulation thresholds of the $\log$-bell shaped function.

5. Main Results. One of the most important features which distinguishes the secondary response from the primary one is that the total antibody level reached in the secondary response is greatly enhanced and the antigen can be eliminated more rapidly. From the results furnished by the simulations, this feature can be observed over a large interval of realistic parameter values. It can be seen that the antibody level in the secondary response is several orders of magnitude higher than that for the primary response. The period of antigen elimination in the secondary response is apparently shortened. Additionally, if 
the antigen concentration becomes higher for the secondary response, the response tends to be stronger. This phenomenon implies that the immune system not only remembers the antigen, but can also eliminate the increased secondary antigen more effectively because of immune memory (Fig. 2). Obviously, the above simulation results are basically consistent with the principle which leads to the vaccination.

Experiments have shown (Maclennan et al., 1990) that in primary response, mainly, $B_{\mathrm{N}}$ cells recruitment and proliferation contribute to raise the antibody level. While during the secondary response, it is $B_{\mathrm{M}}$ cells which dominate the response establishment. The results provided by the present modeling are basically in agreement with those of the experimental observations (Fig. 2). The influences of parameter $\tau$ on the behavior of the model have also been studied. Parameter $\tau$ indicates the moment at which the antigen is introduced for the secondary time. We found, when $\tau$ is relatively small, the introduced antigen can be eliminated rapidly by the large amount of antibodies produced in the primary response. Since in this case the $B_{\mathrm{N}}$ and $B_{\mathrm{M}}$ cells have little chance to receive the stimulation from the antigen, only a small number can be activated to secrete antibodies. Thus the secondary response would be relatively weak. When $\tau$ is relatively large, i.e. after the antibody level being raised in the primary response has declined to an ordinary level, the secondary response is strongly elicited to eliminate the antigen. In this case, $B_{\mathrm{N}}$ and $B_{\mathrm{M}}$ cells have a chance to interact with antigen. It is they that secrete a large number of antibodies which mainly contribute to the secondary response. So there is a threshold of $\tau$, i.e. $\tau_{0}$. When $\tau>\tau_{0}$, the immune system can show an obvious secondary response (see Fig. 3).

Parameters $P_{\mathrm{M}}$ and $S_{\mathrm{M}}$ are the proliferation rate and antibody secretion rate of memory $\mathrm{B}$ cells, respectively. It is these two parameters that distinguish memory B cells from normal B cells. In our modeling, it is found that these two parameters play an important role in the secondary response, i.e. the larger the values of these two parameters, the higher the antibody level the system can reach in the secondary response (see Fig. 4).

The model is set up on the basis of the long-lived clone hypothesis and its distinct feature is that $B_{\mathrm{N}}$ cells and $B_{\mathrm{M}}$ cells have the same or approximately the same lifespan $\left(d_{\mathrm{M}}-d_{\mathrm{N}}-0.5 \mathrm{day}^{-1}\right)$. The problem here is whether we can exclude the long-lived clone hypothesis from the long-lived memory B cells hypothesis. It is estimated that memory B cells may have an average lifespan of 4-6 weeks (Gray and Skarvall, 1988, p. 71; Gray and Leanderson, 1990, p. 9). So we especially set $d_{\mathrm{M}}=0.05$ day ${ }^{1}$ and 0.01 day ${ }^{1}$, corresponding to individual $\mathrm{B}_{\mathrm{M}}$ cell lifespans of 20 and 100 days, respectively. The simulation results show that in the long-lived clone, an individual $B_{\mathrm{M}}$ cell cannot have a long lifespan. Otherwise, $B_{\mathrm{M}}$ cells levels may stay very high for a long time even after antigen has been eliminated in the secondary response. Biologically, we 


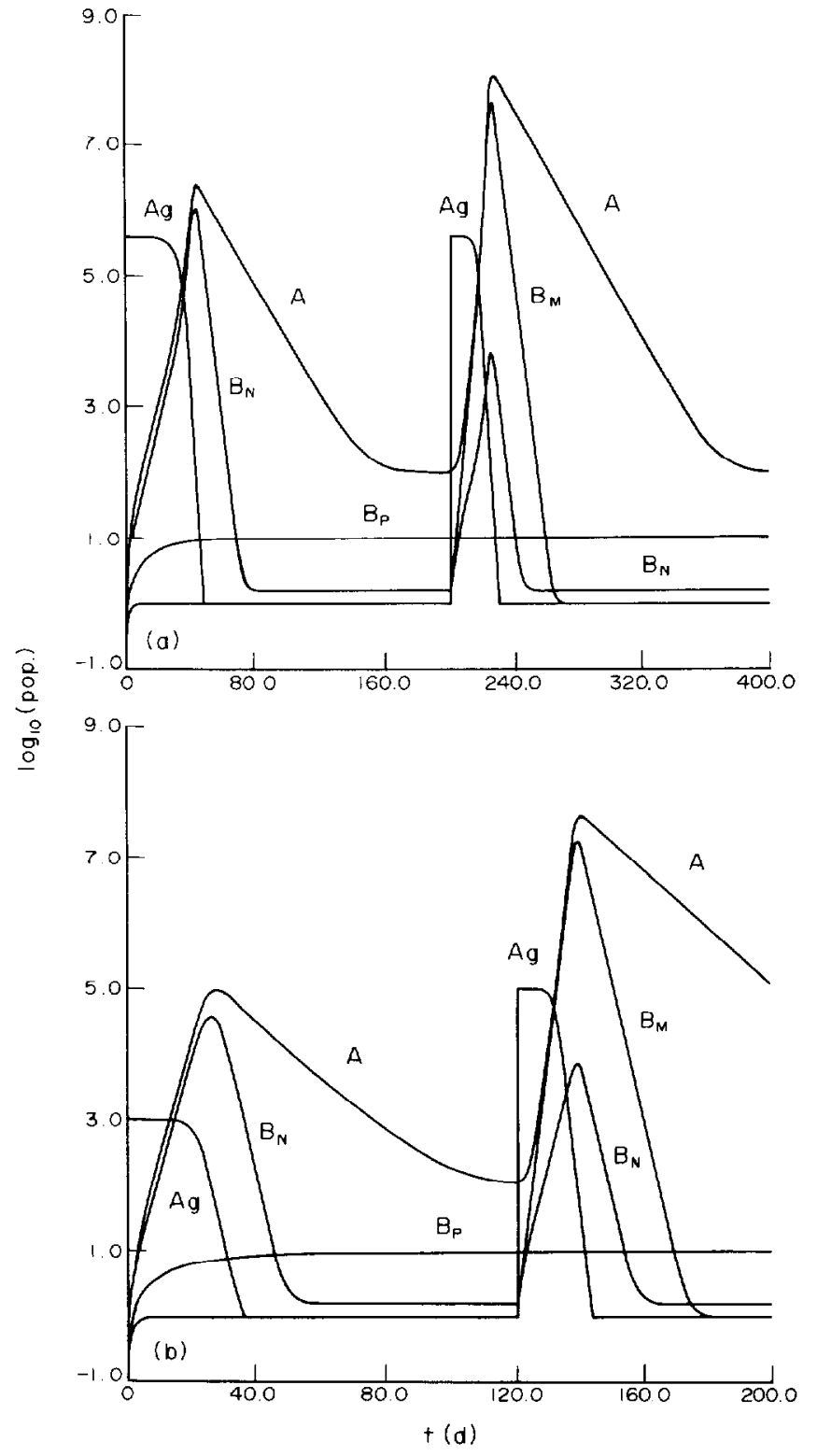

Figure 2. The fundamental features of the secondary response. $d_{\mathrm{A}}=0.1, d_{\mathrm{M}}=d_{\mathrm{N}}=$ $0.5, d_{\mathrm{P}}=0.05, d_{\mathrm{c}}=10^{-3}, P_{\mathrm{N}}=1.0, P_{\mathrm{M}}=1.5, S_{\mathrm{N}}=1.0, S_{\mathrm{M}}=2.0$. (a) For the secondary response, the antibody level is higher than that of the first one with one or more orders of magnitude; the time period for the antibody level reaching its maximum would be much shorter and antigens would be eliminated much faster than them during the first response. $A g 1=A g 2=4 \times 10^{5}, \tau=200$. (b) More antigens are introduced into the individual and it is seen that the secondary response becomes more efficient. $A g 1=10^{3}, A g 2=10^{5}, \tau=120$. In both (a) and (b), The number of $B_{\mathrm{N}}$ is predominant in the primary response while the number of $B_{\mathrm{M}}$ is predominant in the secondary response. 


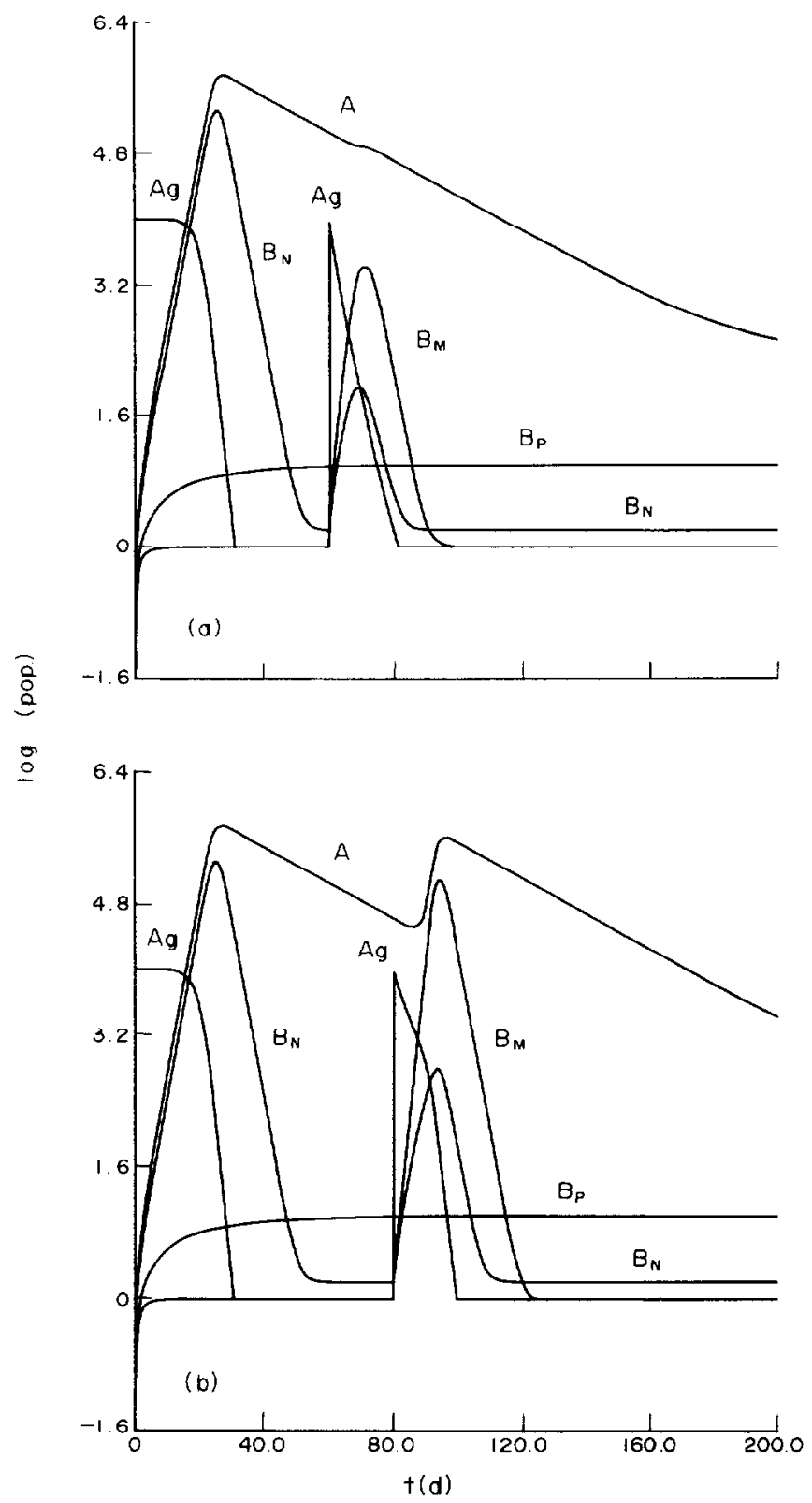

Figure. 3. (a) and (b).

think this seems unreasonable. Thus the long-lived clone hypothesis seems to exclude the long-lived resting $B_{\mathrm{M}}$ cells hypothesis. However, we think the latter remains an alternative to immune memory (Fig. 5).

According to experimental observations, it is known that the antibody 


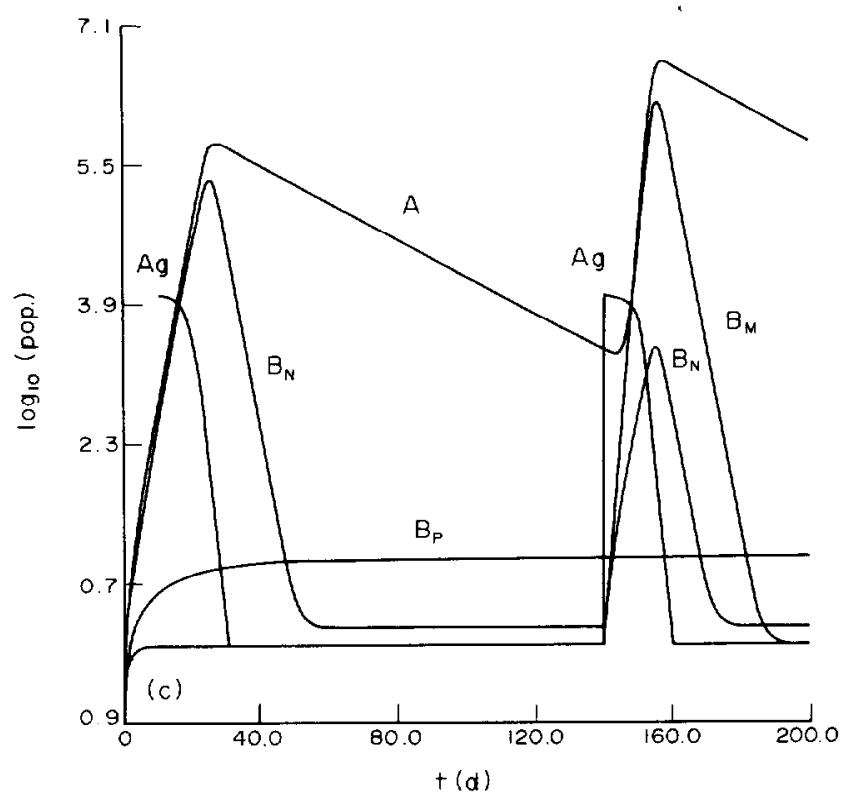

Figure 3. (c).

Figure 3. The influences of the time interval $t$ between the first and the secondary invasions of the antigen on the secondary response, $d_{\mathrm{A}}=0.05, A g 1=A g 2=10^{4}$, $\tau=60,80,140$ in (a), (b) and (c), respectively. (a) and (b) If $\tau<\tau_{0}$, no obvious secondary response can be found and the antigen invaded for the second time would be eliminated fastly owing to the high antibody level primarily activated. (c) When $\tau>\tau_{0}$, the secondary response reappears.

generated in the secondary response has a higher average affinity to the antigen. This phenomenon is referred to as the affinity maturation and is regarded as the result of the mutation of $\mathrm{V}$ regions of immunoglobulin. In order to study how affinity maturation affects the behavior of the model, in the routine, we set $J=0.5$ when $t<\tau$ and $J=1$ when $t>\tau$, simply representing a two-fold increase in antibody affinity in the secondary response. The simulations obviously show that affinity maturation accelerates the process of antigen elimination (not shown by the figures).

The long-lived B plasma cells are incorporated into this model. This is consistent with the experimental result that long-lived B plasma cells are generated in follicular response. However, because of the smallness and stable evaluations of $B_{\mathrm{p}}$ in the antigenic response, when the $B_{\mathrm{M}}$ and $B_{\mathrm{N}}$ cells are predominant, the contribution of $B_{\mathrm{p}}$ can be neglected. We further simplify the five-equation model into a four-equation model by neglecting $B_{\mathrm{p}}$ cells. We found that the four-equation model gives approximately similar results to the five-equation model (not shown by the figures). We thus draw our conclusion that the long-lived B plasma cells play an unimportant role in the antigenic 


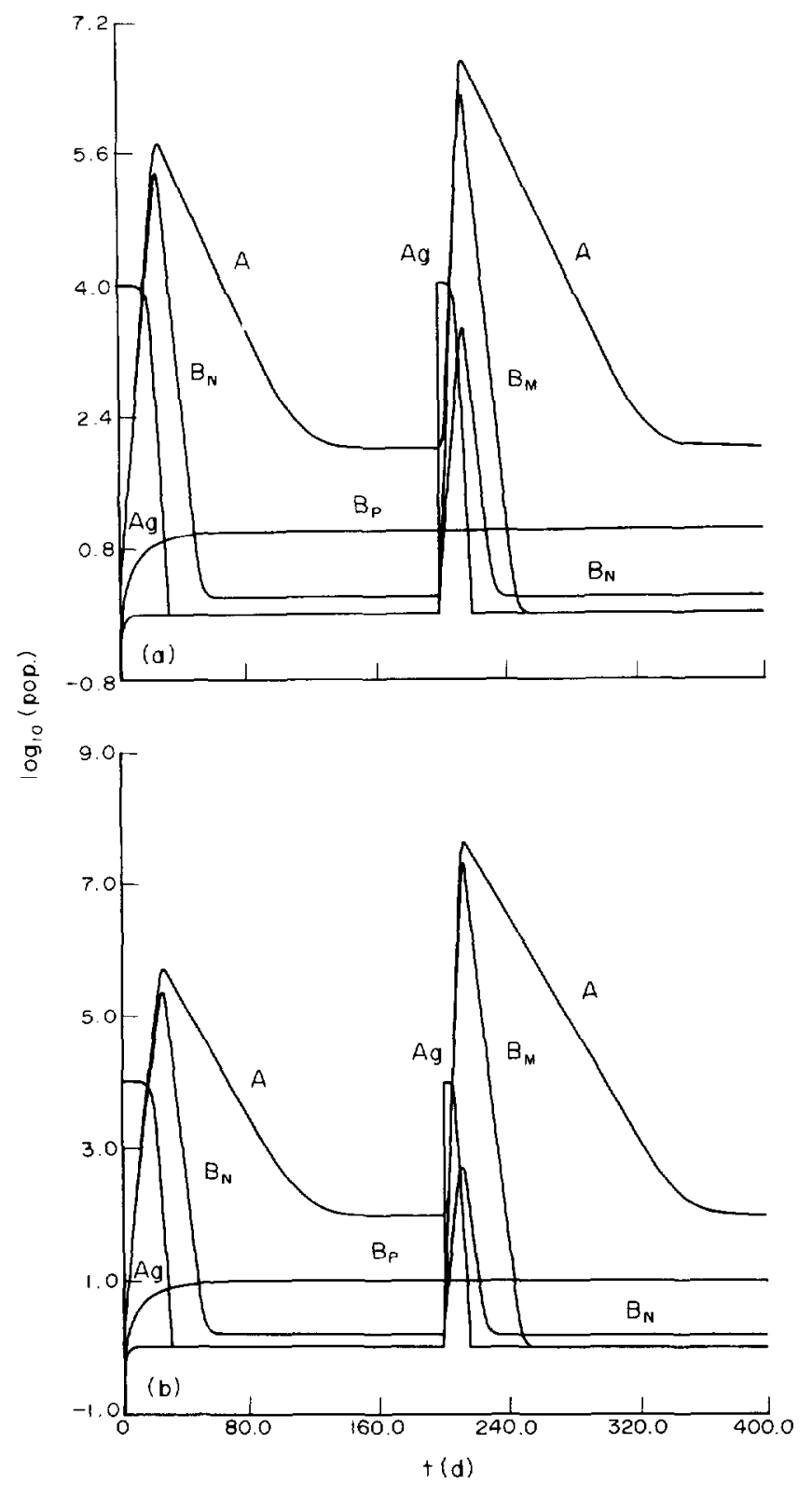

Figure 4. The effections of parameters $P_{\mathrm{M}}$ and $S_{\mathrm{M}}$ on the secondary response. It is found that raising the values of these two parameters is favourable to the secondary response. $d_{\mathrm{A}}=0.1, A g 1=A g 2=10^{4}, \tau=200$. (a) $P_{\mathrm{M}}=1.5$ and $S_{\mathrm{M}}=2.0$. (b) $P_{\mathrm{M}}=2.0$ and $S_{\mathrm{M}}=2.5$. 


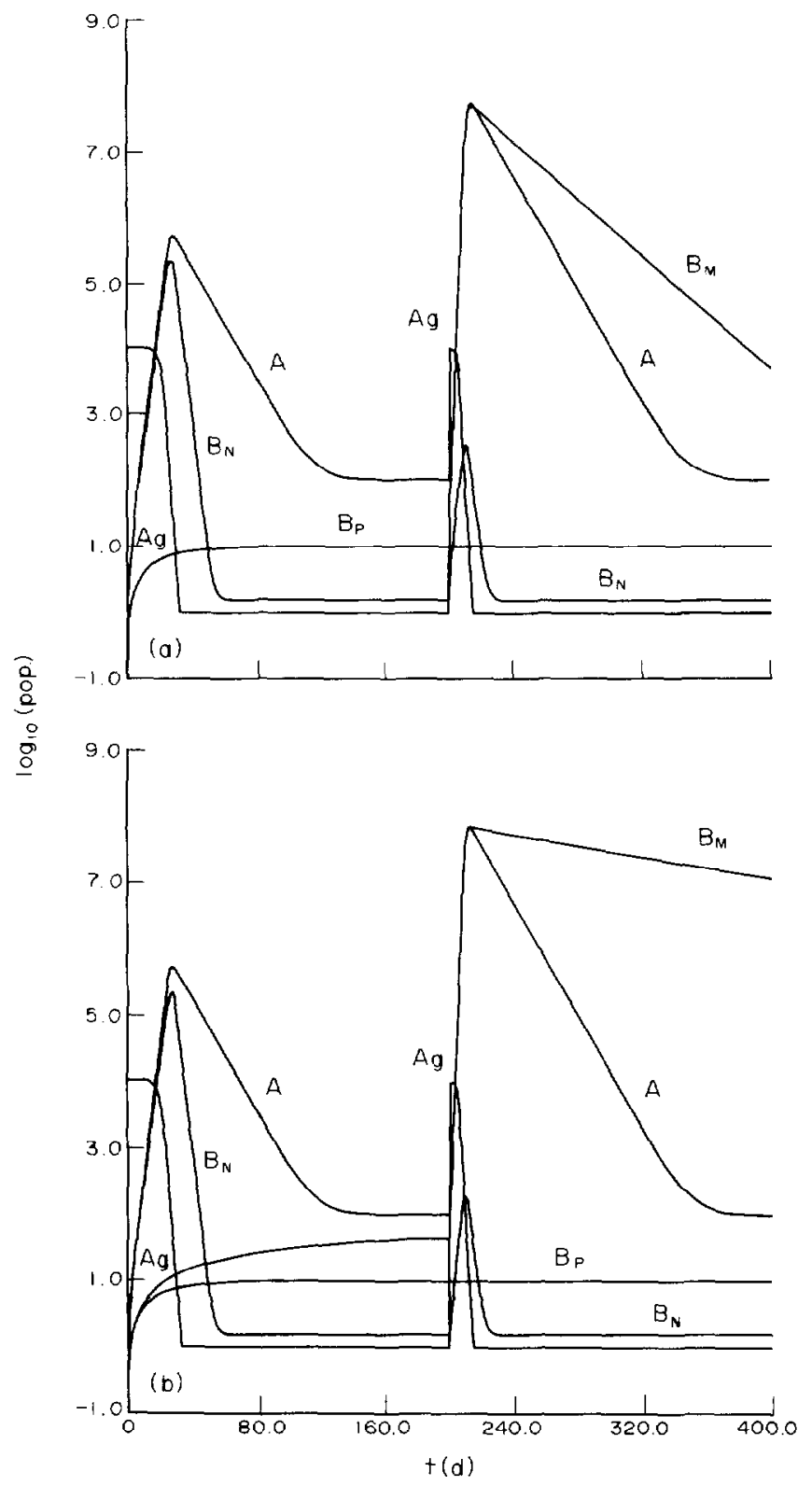

Figure 5. It seems that the existence of long-lived single memory cells is unreasonable. If the lifespan of a single memory cell is too long, the number of $B_{\mathrm{M}}$ cannot reduce to an ordinary level for a long time after the secondary response. The lifespan of a single memory cell is 20 and 100 days for (a) and (b), respectively.

$$
d_{\mathrm{A}}=0.1, A g 1=A g 2=10^{4}, \tau=200 \text {. }
$$


response. Their existence just maintains a certain number of antibodies at ordinary times.

6. Conclusion and Discussion. The first mathematical model that involved memory B cells was constructed by Bell in 1970 (Bell, 1970). The model incorporates four kinds of cells, i.e. target cells, proliferating cells, plasma cells and memory cells. In this note, we set up a simple mathematical model involving normal B cells, memory B cells and long-lived plasma cells to study the contributions of memory $B$ cells to the secondary response. To study this problem, one should first assume what causes immune memory according to experiment. In our model, the mechanism of immune memory is assumed to be the long-lived clone hypothesis. With regard to the key question of how the memory B cells are generated, we have noted two views. One is the "unequal division theory" (Williamson et al., 1976) which suggests that virgin B cells, upon stimulation of antigen, undergo unequal divisions giving rise to both plasma cells and memory B cells. Alternatively, Klinman and Linton proposed the "scparatc lineage theory" (Klinman et al., 1974; Klinman and Linton, 1990) which suggests that memory B cells develop from precursor cells which differ from those responsible for the production of primary antibody response and may represent a separate B cell lineage. This view has been supported by their experiments (Linton and Klinman, 1986; Linton et al., 1989; Klinman and Linton, 1988, 1990). In the present model, we accept the first view, but of course we do not exclude the second one. From the above simulations, it can be seen that the modeling reflects the basic features of the primary and secondary immune response. The dynamical behaviors of the model show that memory B cells indeed play an important role in secondary response.

In a real immune system, the majority of its lymphocytes and antibodies have a very short lifespan. Normally, $\mathbf{B}$ cells have a lifespan about several days, antibodies have a lifespan of about 10 days. How these short-lived components can work together to generate long-term memory seems puzzling. The longlived memory B cells hypothesis gives a solution to this problem, but this solution still raises difficulties. On the one hand, the lifespan of memory B cells, though longer than that of normal B cells, is much shorter than the period which the immune memory to some specific antigen can maintain. In fact, the immune memory to some antigens can be kept throughout the animal's entire lifespan. On the other hand, how memory B cells can alter their lifespan to be long-lived is still a question to be answered (Gray, 1990). The "long-lived clone" hypothesis gives a more realistic path leading to immune memory. It is biologically consistent with the fact that the component members of the immune system are short-lived, thus the adoption of the hypothesis mentioned above can avoid the difficulty that memory B cells expand their lifespan several orders of magnitude longer. Experimentally, "there is clear evidence that clones 
of memory B cells can persist in vivo over long periods of time, the issue of the long-lived, resting memory cell is still controversial" (Rajewsky, 1990, p. 399). For this reason, we based our model on the "long-lived clone" hypothesis. Our simulation results that memory can be obtained by the long-lived $B_{\mathrm{M}}$ clone even the individual $B_{\mathrm{M}}$ cell in this clone is not long-lived provides theoretical support to this hypothesis.

In our model, it seems that the memory to the antigen can be kept forever. This is inconsistent with the fact that immune systems also have "immune forgetting", i.e. memory may be lost after a period of time. We ascribe this the fact that the number of memory $B$ cells in our model is at their equilibrium state at normal times, i.e. at the balance state between the source and the death. In real immune systems, the number of memory B cells should decline as the sequestered antigen is consumed and in this way the "immune forgetting' phenomenon can be explained.

This paper has studied the basic features of the contributions of the memory $B$ cells to the secondary response. In fact, many other kinds of molecules, immune cells and idiotypic network regulation, which also take part in the immune memory, are not taken into account in this paper. For example, Behn et al. (1993) recently studied a model incorporating both idiotypic network and memory $\mathbf{B}$ cells. We noticed that the idiotypic network hypothesis and the memory B cells hypothesis may not be exclusive. Further studies of these factors are in progress on the basis of the modeling studied above.

We thank the referees for their helpful suggestions and constructive comments.

\section{REFERENCES}

Antia, R., B. Levin and P. Williamson. 1991. A quantitative model suggests immune memory involves the colocalization of B and Th cells. I. ther. Biol. 153, 371-384.

Behn, U., J. L. V. Hemmen and B. Sulzer. 1993. Memory to antigenic challenge of the immune system: synergy of idiotypic interactions and memory B cells. $J$. theor. Biol. 165, 1-25.

Bell, G. 1970. Mathematical model of clonal selection and antibody production. J. theor. Biol. 29, 191-232.

Berek, C., J. M. Jarvis and C. Milstein. 1987. Activation of memory and virgin B cell clones in hyperimmune animals. Eur. J. Immunol. 17, 1121-1129.

Coico, R. F., B. S. Bhogal and G. J. Thorbecke. 1983. Relations of germinal centers in lymphoid tissue to immunologic memory. VI. Transfer of B cell memory with lymph node cells fractionated according to their receptors for peanut agglutinin. J. Immunol. 131, 2254-2257.

Coffman, R. L. et al. 1977 . The class of surface immunoglobulin on virgin and memory $\mathbf{B}$ lymphocytes. $J$. Immunol. 118, 1806-1815.

De Boer, R. J. 1988. Symmetric idiotypic networks: connectance and switching, stability, and suppression. In Theoretical Immunology, part two, SFI Studies in the Sciences of Complexity, A. S. Perelson (Ed.). Reading, MA: Addison-Wesley.

De Boer. R. J. and P. Hogeweg. 1989a. Memory but no suppression in low-dimensional symmetric idiotypic networks. Bull. math. Biol. 51, 223-246. 
De Boer, R. J. and P. Hogeweg. 1989b. Unreasonable implications of reasonable idiotypic network assumptions. Bull. math. Biol. 51, 381-408.

De Boer, R. J., I. G. Kevrekidis and A. S. Perelson. 1990. A simple idiotypic network model with complex dynamics. Chem. Eng. Sci. 45, 2375-2382.

De Boer, R. J. and A. S. Perelson. 1991. Size and conectivity as emergent properties of a developing immune network. J. theor. Biol. 149, 381- 424.

Gray, D. 1990. Preface. In Immunological Memory. Berlin: Springer-Verlag.

Gray, D., I. C. M. Maclennan and P. J. L. Lane. 1986. Virgin B cell recruitment and the lifespan of memory clones during antibody responses to 2,4-dinitrophenyl-hemocyanin. Eur. $J$. Immunol. 16, 641-648.

Gray, D. and H. Skarvall. 1988. B-cell memory is short-lived in the absence of antigen. Nature. Lond. 336, 7073

Gray, D. and T. Leanderson. 1990. Expansion, selection and maintenance of memory B-cell clones. In Immunological Memory. Gray, D. (Ed.). Berlin: Springer-Verlag.

Ho, F., J. E. Lortan, I. C. M. Maclennan and M. Khan. 1986. Distinct short-lived and long-lived antibody-producing cell populations. Eur. J. Immunol. 16, 1297-1301.

Hood, L. E. et al. 1984. In Immunology. 2nd edn, p. 11. Menlo Park, CA: Benjamin Cummings.

Jerne, N. K. 1974. Towards a network theory of the immune system. Ann. Immunol. (Inst. Pasteur) 125c, 373-389.

Jerne, N. K. 1984. Idiotypic network and other preconceived ideas. Immunol. Rev. 79, 5-24.

Kaufman, M., J. Urbain and R. Thomas. 1985. Towards a logical analysis of the immune response. $J$. theor. Biol. 114, 527-561.

Kepler, T. B. and A. S. Perelson. 1993. Somatic hypermutation in B cells: an optimal control treatment. I. theor. Biol 164, 37-64.

Klaus, G. G. B. 1978. The generation of memory cells. II. Generation of B memory cells with preformed antigen-antibody complexes. Immunology 34, 643-652.

Klinman, N. R. and P. J. Linton. 1988. The clonotype repertoire of B cell subpopulations. Adv. Immunol. 42, 1-93.

Klinman, N. R. and P. J. Linton. 1990. The generation of B-cell memory: a working hypothesis. In Immunological Memory. pp. 19-33. Berlin: Springer-Verlag.

Klinman, N. R., J. L. Press, A. R. Pickard, R. T. Woodland and A. F. Dewey. 1974. Biography of the B cell. In The immune System. E. Sercarz, A. Williamson and C. F. Fox (Eds), pp. 357-365. New York: Academic Press.

Lane, P. J. L., D. Gray, S. Oldfield and I. C. M. Maclennan. 1986. Differences in the recruitment of virgin B cells into antibody responses to thymus-dependent and thymus-independent type-2 antigens. Eur. J. Immunol. 16, 1569-1575.

Levy, M. and A. Coutinho. 1987. Long-lived B cells: mitogen activity as a tool for studying their life-spans. Eur. J. Immunol. 17, 295-298.

Linton, P. J. and N. R. Klinman. 1986. The generation of secondary B cells in vitro (abstract). Fed. Proc. 45, 378.

Iinton, P. I., D. J. Decker and N. R. Klinman. 1989. Primary antibody-forming cells and secondary B cells are generated from separate precursor cell subpopulations. Cell $\mathbf{5 9}$, $1049-1059$.

Liu, Y-J., S. Oldfield and I. C. M. Maclennan. 1988. Memory B cells in T cell-dependent antibody responses colonize the splenic marginal zones. Eur. J. Immunol. 18, 355-362.

Liu, Y. J., D. E. Joshua, G. T. Williams, C. A. Smith, J. Gordon and I. C. M. Maclennan. 1989. Mechanism of antigen-driven selection in germinal centres. Nature 342, 929-931.

Maclennan, I. C. M., Y-J. Liu, D. E. Joshua and D. Gray. 1989. The production and selection of memory B cells in follicles. In Progress in Immunology. VII, F. Melchers et al. (Eds). Berlin: Springer-Verlag.

Maclennan, I. C. M., Y-J. Liu, S. Oldfield, J. Zhang and P. J. L. Lane. 1990. The evolution of B cell clones. In Immunological Memory, pp. 1-14. Berlin: Springer-Verlag.

Mandel, T. E., R. P. Phipps, A. Abbot and J. G. Tew. 1980. The follicular dendritic cell: long term antigen retention during immunity. Immunol. Rev. 53, $29-59$. 
Mandel, T. E., R. P. Phipps, A. Abbot and J. G. Tew. 1981. Long term antigen retention by dendritic cells in the popteal lymph nodes of immunized mice. Immunology 43, 353-362.

Parisi, G., 1990. A simple model for the immune network. Proc. natn. Acad. Sci. U.S.A. 87, 429-433.

Perelson, A. S. and C. Delisi. 1980. Receptor clustering on a cell surface. I. Theory of receptor cross-linking by ligands bearing two chemically identical functional groups. Math. Biosci.48, $71-110$.

Perelson, A. S. 1988. Towards a realistic model of the immune system. In Theoretical Immunology, part two, SFI Studies in the Sciences of Complexity, A. S. Perelson, (Ed.). Reading, MA: Addison-Wesley.

Perelson, A. S. 1989. Immune network theory. Immunol. Rev. 110, 5-36.

Perelson, A. S. and G. Weisbuch. 1992. Modeling immune reactivity in secondary lymphoid organs. Bull. math. Biol. 54, 649-672.

Rajewsky, K. 1989. Evolutionary and somatic immunological memory. In Progress in Immunology. VII, F. Melchers et al. (Eds). Berlin: Springer-Verlag.

Sulzer, B., J. Leo Van Hemmen, A. U. Neumann and U. Behn. 1993. Memory in idiotypic networks due to competition between proliferation and differentiation. Bull. math. Biol.55, $1132-1182$.

Tew, J. G. and T. E. Mandel. 1978. The maintenance and regulation of serum antibody level: evidence indicating a role for antigen retained in lymphoid follicles. J. Immunol. 120, $1063-1069$.

Tcw, J. G. and T. E. Mandel. 1979. Prolonged antigen half-life in the lymphoid follicles of specifically immunized mice. Immunology 37, 69-76.

Weisbuch, G., R. J. De Boer and A. S. Perelson. 1990. Localized memory in idiotypic networks. $J$. theor. Biol. 146, 483-499.

Williamson, A. R., I. M. Zitron and A. J. McMichael. 1976. Clines of B lymphocytes: their natural selection and expansion. Fed. Proc. 35, 2195-2201.

Yefenof, E. et al. 1985. Preparation and analysis of antigen-specific memory B cells. J. Immunol. 135, 3777 .

Received 24 January 1994

Revised version accepted 1 February 1995 